\title{
TRANSPORTE E CARACTERIZAÇÃO DE SEDIMENTOS DE FUNDO NO RIO PIRANHAS EM UMA SEÇÃO DE CONTROLE PRÓXIMO À SEDE DO MUNICÍPIO DE POMBAL-PB
}

\author{
D. L. CRISPIM ${ }^{1 *}$, L. L. ISMAEL ${ }^{2}$, T. M. I. DE SOUSA ${ }^{2}$, J. W. A. GARRIDO ${ }^{3}$ e M. M. F. DE QUEIROZ ${ }^{1}$ \\ ${ }^{1}$ Universidade Federal de Campina Grande - UFCG \\ ${ }^{2}$ Universidade Federal da Paraíba - UFPB \\ ${ }^{3}$ Universidade Federal do Rio Grande do Norte - UFRN \\ diegolc_85@hotmail.com*
}

Artigo submetido em outubro/2014 e aceito em maio/2015

DOI: 10.15628/holos.2015.2518

\section{RESUMO}

O Rio Piranhas-Açu constitui um dos principais eixos de drenagem natural da bacia do Piranhas Açu, no nordeste do Brasil. Este rio de domínio federal, uma vez que nasce no município de Bonito de Santa Fé, no Estado da Paraíba, e segue seu curso natural pelo Estado do Rio Grande do Norte, desaguando no Oceano Atlântico, na Costa Potiguar. A fim de caracterizar os sedimentos e mensurar seu transporte pelo Rio Piranhas, a partir de suas características geométricas e de material do leito amostrado, realizou-se análise granulométrica e calculou-se o transporte no leito através de fórmula empírica. A equação utilizada foi a de Meyer-Peter \& Müller (transporte no leito). A equação de Meyer-Peter \& Müller produziu resultados que aparentemente seguem a variação da granulometria dos sedimentos do leito do rio. Equações para a estimativa do transporte de sedimentos no rio podem não ter uma precisão adequada devido a problemas na sua calha fluvial.

PALAVRAS-CHAVE: Hidrometria, Monitoramento Ambiental, Semiárido.

\section{BOTTOM SEDIMENT TRANSPORT AND CHARACTERIZATION IN PIRANHAS RIVER AT A CONTROL SECTION NEAR THE TOWN OF POMBAL-PB}

\begin{abstract}
The Piranhas-Acu River is one of the major routes of natural drainage basin Piranhas Acu. This river of federal domain, once born in the city of Bonito Santa Fe, State of Paraiba, and follows its natural course by the State of Rio Grande do Norte, emptying into the Atlantic Ocean in Costa Potiguar. In order to characterize the sediment and measure their transport by Rio Piranhas, from its geometric characteristics and bed material sampled was held sieve analysis and calculated the transport through
\end{abstract}

the bed of empirical formula. The equation used was that of Meyer-Peter \& Müller (transport in the bed). The equation of Meyer-Peter \& Müller produced results that apparently follow the variation in grain size of the sediment from the riverbed. Equations to estimate sediment transport in the river may not have an adequate accuracy due to problems in your fluvial system.

KEYWORDS: Hydrometric, Environmental Monitoring, semiarid, water quality. 


\section{INTRODUÇÃO}

O termo sedimento refere-se à partícula derivada da fragmentação das rochas, seja por processos físicos ou químicos que é transportada por ação de agentes externos como água e vento, do seu lugar de origem aos rios e locais de deposição. Nos rios estes sedimentos podem ser encontrados principalmente em suspensão na água ou depositado no leito (CARVALHO et al., 2000).

O estudo do transporte de sedimento em rios é importante com relação à poluição da água, navegabilidade do canal do rio, assoreamento de reservatórios, vida útil dos equipamentos de hidroelétricas, habitat dos peixes e outras vidas aquáticas, estética do rio (COBANER \& KISI, 2009).

O conhecimento do comportamento hidrossedimentológico de corpos hídricos é fundamental para a adequada gestão e uso de seus recursos hídricos, bem como para dar suporte à decisão sobre o desenvolvimento de atividades antrópicas, especialmente em áreas de bacias hidrográficas. O acompanhamento dos fluxos de sedimentos ocorrido em um dado corpo hídrico permite ainda o diagnóstico de eventuais impactos em sua área de drenagem ao longo do tempo, podendo se tornar um importante indicador ambiental.

Em várias atividades de aproveitamento dos recursos hídricos de superfície, a concentração e a qualidade dos sedimentos são informações essenciais. Carvalho et al. (2000) afirmam que o transporte de sedimentos afeta a qualidade da água e a possibilidade para o consumo humano ou seu uso para outras finalidades. Numerosos processos industriais não toleram mesmo pequenas porções de sedimentos em suspensão na água. Esse fato envolve muitas vezes enormes gastos públicos para a solução do problema.

Nos últimos anos, os sedimentos têm sido considerados como fonte secundária de poluição, quando partículas contaminadas são mobilizadas e contaminantes são liberados dentro da água, após suspensão natural ou artificial dos sedimentos. Em escala de bacia, informações químicas e ecológicas necessitam de uma forte base de dados quantitativos de sedimentos (APITZ; WHITE, 2003).

Sedimentos fluviais são aqueles que após erodirem, atingem os cursos d'água. Nos rios o movimento dos sedimentos apresenta-se de duas formas: o transporte de sedimentos em suspensão e transporte no leito. O transporte em suspensão é constituído por partículas mais finas, mantidas distantes do leito do canal pelo movimento da água (turbulência) e somente se depositam quando a velocidade do fluido diminui. O transporte no leito, caracteriza-se por ser governado basicamente pela gravidade o que faz com que os sedimentos rolem, saltitem e sejam arrastados pelo fluxo. Por isso, este é o modo de transporte das partículas mais graduadas.

Uma separação clara do transporte sólido em transporte no leito e em suspensão, não existe com rigor, tratando-se de uma idealização das condições reais. De fato, não existe uma fronteira definida a partir da qual se possa distinguir se o caminho dos grãos encontra-se junto ao fundo ou se as partículas prosseguem em suspensão.

A erosão hídrica é o principal agente da degradação dos solos e dos recursos hídricos em ambientes tropicais e subtropicais úmidos, sendo a perda da camada superficial do solo, o maior desafio para a sustentabilidade da agricultura no mundo. Este fenômeno, cuja ocorrência na natureza é própria da evolução da paisagem, ganha proporções gigantescas quando da presença 
de interferência antrópica. Remoção da cobertura vegetal original, agricultura intensiva, desrespeito às leis ambientais e de ordenamento territorial e a não observância da capacidade de uso do solo são alguns dos fatores que contribuem para o processo de degradação. A bacia hidrográfica do rio Piranhas-Açu reflete essa realidade de degradação e suas consequências sobre os recursos hídricos, apresentando situação de degradação em diferentes estágios (ANA, 2011).

$\mathrm{Na}$ bacia do rio Piranhas a medição de sedimentos é feita em pouquíssimos pontos, em duas épocas distintas por ano, junto a estações fluviométricas que medem descargas líquidas diárias o ano inteiro. A bacia do rio Piranhas está inserida na Bacia Hidrográfica do rio PiranhasAçu situada no Nordeste do Brasil, pertencente ao território dos estados do Rio Grande do Norte e da Paraíba, totalmente inserida no clima semiárido nordestino. Possui uma área total de drenagem de $43.681,50 \mathrm{Km}^{2}$, sendo $26.183,00 \mathrm{Km}^{2}$, correspondendo a $60 \%$ da área no Estado da Paraíba, e o restante no Estado do Rio Grande do Norte.

O principal rio da bacia é o rio Piranhas-Açu, de domínio federal, uma vez que nasce no município de Bonito de Santa Fé, no Estado da Paraíba, e segue seu curso natural pelo Estado do Rio Grande do Norte, desaguando no Oceano Atlântico, na Costa Potiguar. O rio é denominado Piranhas no estado da Paraíba e, após cruzar a fronteira com o estado do Rio Grande do Norte é que adquire o nome de Piranhas-Açu. A bacia é constituída por sete sub-bacias: Piancó, Peixe, Alto Piranhas, Médio Piranhas, Espinharas, Seridó e Baixo Piranhas. As três primeiras estão totalmente inseridas em território paraibano, a sub-bacia do Baixo Piranhas situa-se totalmente no estado do Rio Grande do Norte e as demais estão compreendidas nos dois estados.

A parte da bacia do rio Piranhas, constituída pelas sub-bacias: Piancó, Peixe e Alto Piranhas apresenta capacidade de armazenamento de $1.846 .126 .108 \mathrm{~m}^{3}, 143.791 .396 \mathrm{~m}^{3} \mathrm{e}$ $322.513 .781 \mathrm{~m}^{3}$ respectivamente, através de importantes reservatórios como o sistema Coremas-Mãe D’Água, Engenheiro Ávidos, São Gonçalo, Lagoa do arroz, Capivara entre vários outros, isso permite a perenização de trechos dos rios Piancó, do Peixe e do Alto Piranhas e, por conseguinte do rio Piranhas até a montante da barragem Armando Ribeiro Gonçalves, no Estado do Rio Grande do Norte.

Os regimes hidrológicos desses rios sofrem fortes influências das condições climáticas do semiárido. Notadamente, no curto período chuvoso, ocorrido anualmente, onde as variações de vazões, da qualidade da água e da quantidade de sedimentos são decorrentes dos volumes de água superficiais, associadas às formas de uso do solo, às características fisiográficas das bacias e ao tipo de cobertura vegetal predominante da caatinga.

Além disso, devido às características geológicas das citadas bacias, esses rios têm limitações de produção de água, ficando os mesmos com caráter temporários, onde as vazões ocorridas no período chuvoso são resultados praticamente do escoamento superficial, em que sua qualidade fica comprometida pelo aporte de sedimentos, nutrientes e agroquímicos. Já no período de estiagem a vazão regularizada pelos reservatórios tem sua qualidade comprometida pelo aporte de águas servidas de uso doméstico e agrícola.

O aporte de sedimentos nessas bacias fica condicionado ao período chuvoso, com características bem peculiares as condições do semiárido, em que no início das chuvas a caatinga encontra-se sem folhagem, deixando o solo exposto à ação da erosão da chuva, principal causa da degradação dos solos e dos recursos hídricos em ambientes tropicais. 
O presente trabalho teve como finalidade executar um estudo sobre o transporte de sedimentos de fundo na bacia hidrográfica do rio Piranhas, sub-bacia do Piranhas-Açu, definida a partir da secção de controle localizada junto à ponte da BR 230 sobre o citado rio, sob as coordenadas: $-6: 43: 43$ de latitude e -37:47:40 de longitude.

\section{MATERIAL E MÉTODOS}

\section{1 Área de estudo}

O presente estudo foi executado na bacia hidrográfica do rio Piranhas, na secção de controle junto a Ponte sobre o rio Piranhas, na BR 230, distante $5 \mathrm{~km}$ da cidade de Pombal-PB (Figura 1).

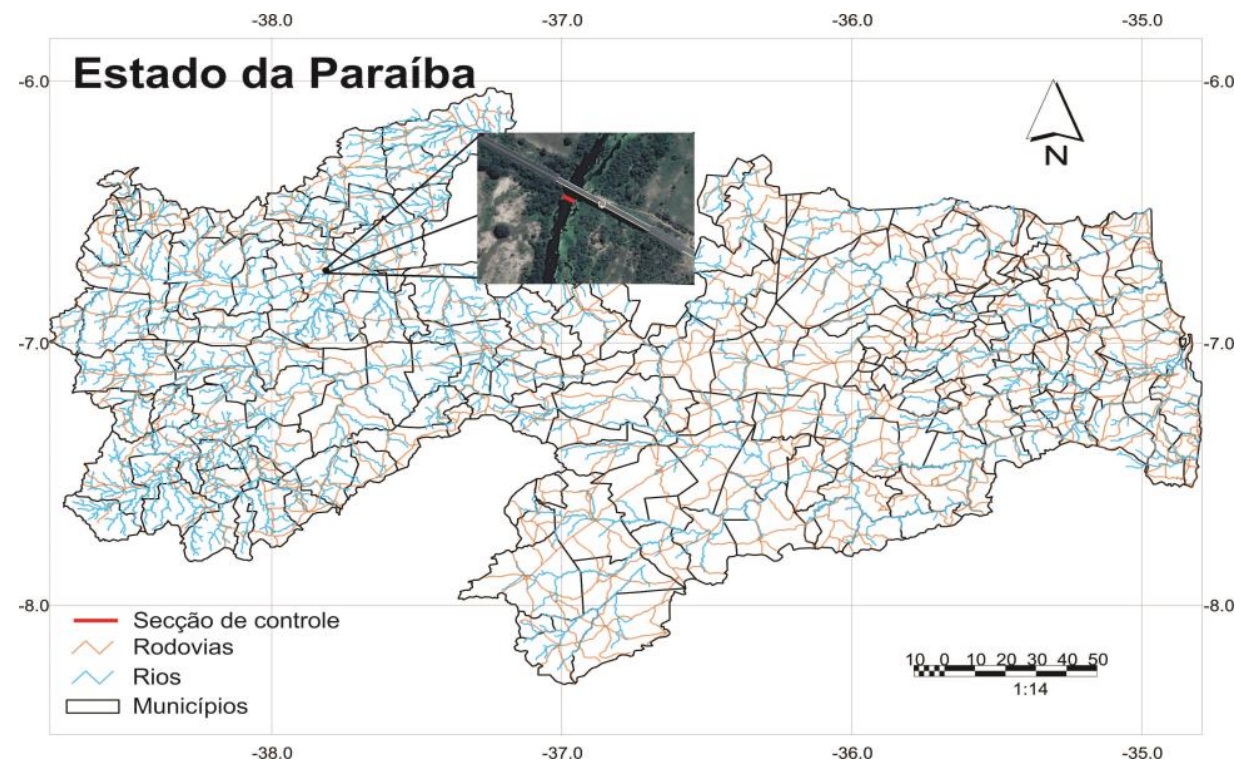

Figura 1: Imagem da secção de controle junto a Ponte sobre o rio Piranhas, na BR 230. Fonte: Autor.

Na secção de controle da bacia foram realizadas no período de 06/03/2012 a 22/06/2012 oito medições da descarga líquida e de sedimentos de fundo. A vazão foi determinada através do método velocidade-área da meia seção, em que a velocidade de fluxo foi medida através de molinete fluviométrico com contador eletrônico de pulso (Figura 2) e através de medidor acústico Doppler de vazão, usando o ADP RiverSurveuor S5 da Sontek.

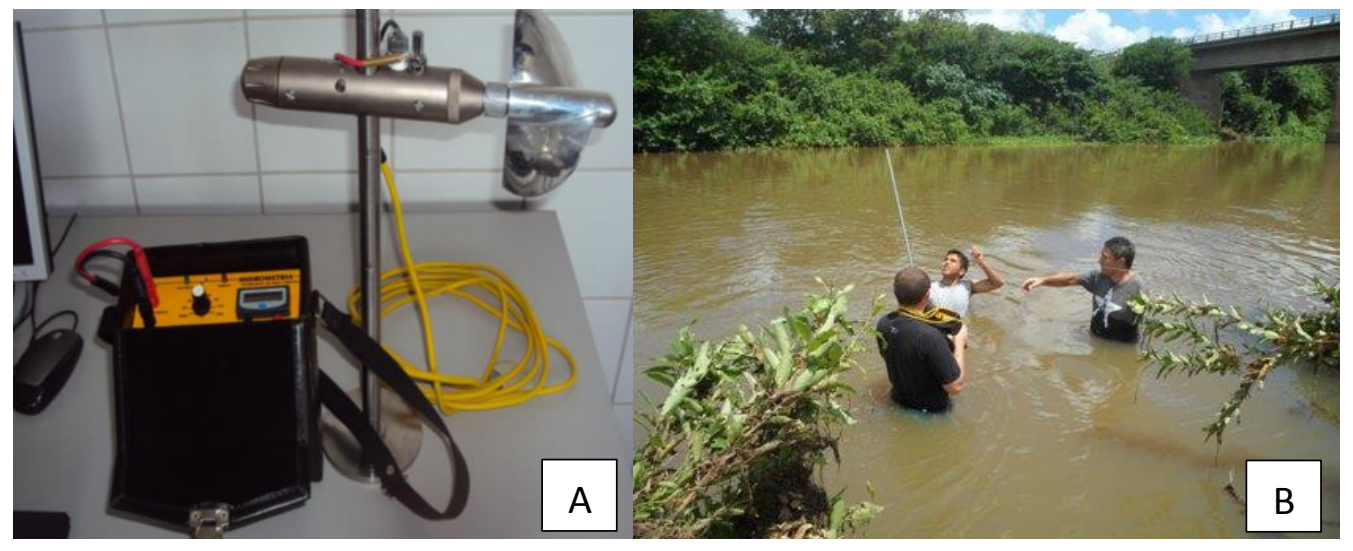

Figura 2: A. Molinete fluviométrico. B. Determinação da velocidade do fluxo de água no rio Piranhas, Pombal-PB (2012). 
As amostras de sedimentos de fundo foram obtidas durante o processo de medição da vazão, em pontos coincidentes com as verticais da batimetria, usando o amostrador USBM-54 e a draga de Peterson, as quais eram misturadas configurando amostras compostas. No processo de amostragem fez-se uso do barco de alumínio, com guincho, motor de popa e lastro fluviométrico. Esses equipamentos se encontram disponíveis no laboratório de Hidrologia da UFCG/Campus Pombal-PB.

\subsection{Análise de Sedimento de Fundo - Granulometria}

Para avaliação do material do leito, utiliza-se a análise granulométrica para identificar o tamanho das partículas ou grãos, e a sua distribuição em porcentagem dos diâmetros dos grãos. Desta análise determina-se o tamanho e a graduação das partículas constituintes do solo. Para a realização dos ensaios, as amostras da seção estudada, são secadas na estufa e posteriormente desagregam-se os torrões. Em seguida, é feito a separação para obter-se uma amostra representativa. Após a separação, foi realizada a classificação granulométrica com a utilização de peneiras de números: $5,10,18,35,60,80,120$, e 230 . Os quais forneceram os seguintes diâmetros característicos: D90, D84, D65, D50, D35, D16.

\subsection{Cálculo da descarga sólida}

Para o cálculo de suas características geométricas da seção em cada campanha foram calculados $y$, a profundidade em cada segmento, $(A)$ área, $(P)$ perímetro molhado e $(R h)$ o raio hidráulico.

Devido a necessidade de estimar a declividade i, do fundo do rio nas seções (canal principal), a equação de Manning-Strickler foi utilizada. $O$ uso desta equação requer, além dos dados geométricos da seção, do coeficiente de rugosidade de Manning, o qual foi calculado a partir da granulometria dos sedimentos do leito com as equações 1 e 2 . 0 valor médio encontrado com as duas fórmulas foi o adotado para cada seção considerada.

$$
\begin{gathered}
n=\frac{d_{50} 1 / 6}{21,1} \\
n=\frac{d_{90}{ }^{1 / 6}}{26,0} \\
I=\left(\frac{Q n}{A R h^{2 / 3}}\right)^{2}
\end{gathered}
$$

Em que:

$\mathrm{n}$ - coeficiente de rugosidade de Manning.

$d_{50}$ - diâmetro da partícula para qual $50 \%$ do sedimento de leito são mais finos $-m$

$\mathrm{d}_{90}$ - diâmetro da partícula para qual $90 \%$ do sedimento de leito são mais finos $-m$

$\mathrm{Q}$ - vazão $-\mathrm{m}^{3} / \mathrm{s}$

A - área molhada da secção transversal $-m^{2}$

$\mathrm{Rh}$ - raio hidráulico - $\mathrm{m}$ 


\subsection{Método Meyer-Peter \& Muller: transporte no leito}

A Fórmula de Meyer-Peter e Müller fornece a taxa de sedimentos transportados por metro de largura das seções (CARVALHO, 2008).

$$
\begin{gathered}
\gamma \frac{Q_{s}}{Q}\left[\frac{k_{s}}{k_{r}}\right]^{3 / 2} p I=0,047\left(\gamma_{s}-\gamma\right) D_{m}+0,25\left[\frac{\gamma}{g}\right]^{1 / 3} q_{s a}^{2 / 3} \\
k_{r}=\frac{26}{D_{90}^{1 / 6}}
\end{gathered}
$$

Em que:

$\gamma$ - peso específico da água $-t / m^{3}$

Qs - parte da descarga líquida que influencia no leito - L/s

$\mathrm{Q}$ - descarga líquida total - L/s

Ks - coeficiente de rugosidade do leito de Strickler igual ao inverso do coeficiente de Manning

$\mathrm{Kr}$ - coeficiente de rugosidade das partículas $-\mathrm{m}^{1 / 3} / \mathrm{s}$

$D_{90}$ - diâmetro da partícula para qual $90 \%$ do sedimento de leito são mais finos - $m$

$\mathrm{P}$ - profundidade média - $\mathrm{m}$

1 - gradiente de energia $-\mathrm{m} / \mathrm{m}$

$\gamma_{s}$ - peso específico do sedimento igual a $2,65 \mathrm{t} / \mathrm{m}^{3}$

$\mathrm{D}_{\mathrm{m}}$ - diâmetro médio da composição do material de leito

$\mathrm{g}$ - aceleração da gravidade igual a $9,815 \mathrm{~m} / \mathrm{s}^{2}$

qsa - descarga sólida do leito - t/s por $m$ de largura

\section{RESULTADOS E DISCUSSÃO}

No decorrer da pesquisa, foram realizadas 8 campanhas de medições hidrossedimentométricas, que abrange as medições de descarga líquida e sólida na seção de controle do rio Piranhas- PB. Na Tabela 1 são apresentados os parâmetros hidráulicos e os valores de vazão obtidos nas oito campanhas na secção de controle.

Tabela 1: Parâmetros Hidráulicos e os valores de vazão

\begin{tabular}{c|c|c|c|c|c|c}
\hline Data das campanhas & Lt & Ph & Am & Pm & Rh & Q \\
\hline $06 / 03 / 2012$ & 45,00 & 0,68 & 30,44 & 45,33 & 0,67 & 9,73 \\
\hline $12 / 03 / 2012$ & 44,00 & 0,68 & 29,90 & 44,35 & 0,67 & 7,32 \\
\hline $20 / 03 / 2012$ & 39,67 & 0,59 & 23,40 & 39,99 & 0,59 & 4,73 \\
\hline $10 / 04 / 2012$ & 39,78 & 0,64 & 25,40 & 40,10 & 0,63 & 4,32 \\
\hline $23 / 05 / 2012$ & 39,48 & 0,64 & 25,40 & 39,80 & 0,64 & 4,02 \\
\hline $30 / 05 / 2012$ & 40,70 & 0,63 & 25,80 & 41,03 & 0,63 & 4,31 \\
\hline $10 / 06 / 2012$ & 41,27 & 0,63 & 26,20 & 41,60 & 0,63 & 4,33 \\
\hline $22 / 06 / 2012$ & 41,90 & 0,64 & 26,90 & 42,24 & 0,64 & 4,36 \\
\hline
\end{tabular}

L: Largura do topo $(\mathrm{m})$; Profundidade hidráulica $(\mathrm{m})$; Área molhada $\left(\mathrm{m}^{2}\right)$; Perímetro molhado (m); Raio hidráulico (m); Vazão $\left(\mathrm{m}^{3} / \mathrm{s}\right)$. 
A distribuição granulométrica do material de leito, referente as oito campanhas estão apresentadas na Tabela 2.

Tabela 2: Porcentagens da fração de grãos do material do leito do rio Piranhas retido

\begin{tabular}{c|c|c|c|c|c|c|c|c}
\hline Data & $\mathbf{D ( 5 )}$ & $\mathbf{D ( 1 0 )}$ & $\mathbf{D}(\mathbf{1 8})$ & $\mathbf{D}(\mathbf{3 5})$ & $\mathbf{D}(\mathbf{6 0})$ & $\mathbf{D}(\mathbf{8 0})$ & $\mathbf{D}(\mathbf{1 2 0})$ & $\mathbf{D}(\mathbf{2 3 0})$ \\
\hline $06 / 03 / 2012$ & 23,38 & 27,18 & 16,61 & 17,13 & 11,29 & 0,50 & 2,26 & 1,65 \\
\hline $12 / 03 / 2012$ & 3,55 & 2,19 & 10,61 & 36,08 & 29,95 & 1,49 & 10,86 & 5,27 \\
\hline $20 / 03 / 2012$ & 3,25 & 12,18 & 16,40 & 23,75 & 32,56 & 3,26 & 6,88 & 1,72 \\
\hline $10 / 04 / 2012$ & 3,72 & 10,12 & 23,52 & 32,80 & 20,20 & 2,94 & 5,21 & 1,49 \\
\hline $23 / 05 / 2012$ & 5,73 & 11,35 & 17,50 & 28,92 & 22,71 & 4,65 & 7,21 & 1,93 \\
\hline $30 / 05 / 2012$ & 18,72 & 24,38 & 18,33 & 20,40 & 14,71 & 0,68 & 1,78 & 1,00 \\
\hline $10 / 06 / 2012$ & 21,49 & 26,19 & 18,11 & 19,30 & 9,42 & 3,52 & 1,63 & 0,34 \\
\hline $22 / 06 / 2012$ & 24,27 & 22,48 & 19,12 & 18,73 & 12,74 & 1,02 & 1,22 & 0,42 \\
\hline
\end{tabular}

Em que: $\mathrm{D}(5)$ : diâmetro passante na peneira no 5 com abertura de malha correspondente a $4 \mathrm{~mm}$; $\mathrm{D}(10)$ : diâmetro passante na peneira no $10 \mathrm{com}$ abertura de malha correspondente a $2 \mathrm{~mm}$; $\mathrm{D}(18)$ : diâmetro passante na peneira no $18 \mathrm{com}$ abertura de malha correspondente a $1 \mathrm{~mm}$; $\mathrm{D}(35)$ : diâmetro passante na peneira $\mathrm{n}$ o 35 com abertura de malha correspondente a $0,5 \mathrm{~mm}$. $\mathrm{D}(60)$ : diâmetro passante na peneira $\mathrm{n}$ - $60 \mathrm{com}$ abertura de malha correspondente a 0,25mm; $D(80)$ : diâmetro passante na peneira no 80 com abertura de malha correspondente a 0,177mm; $D(120)$ : diâmetro passante na peneira no 120 com abertura de malha correspondente a $0,125 \mathrm{~mm}$; $D(230)$ : diâmetro passante na peneira $\mathrm{n} 0230$ com abertura de malha correspondente a $0,062 \mathrm{~mm}$.

A partir dos dados referentes à distribuição granulométrica do material de leito apresentado na Tabela 2 confeccionaram-se as curvas granulométricas do material de leito das oito campanhas, exibidas na Figura 3.

De acordo com a classificação granulométrica da Associação Brasileira de Normas Técnicas - ABNT (norma NBR 6.502/1995), citada por Carvalho (2008) o tipo de sedimento que está em contato com o leito do rio Piranhas, teve variação de pedregulho a areia fina.

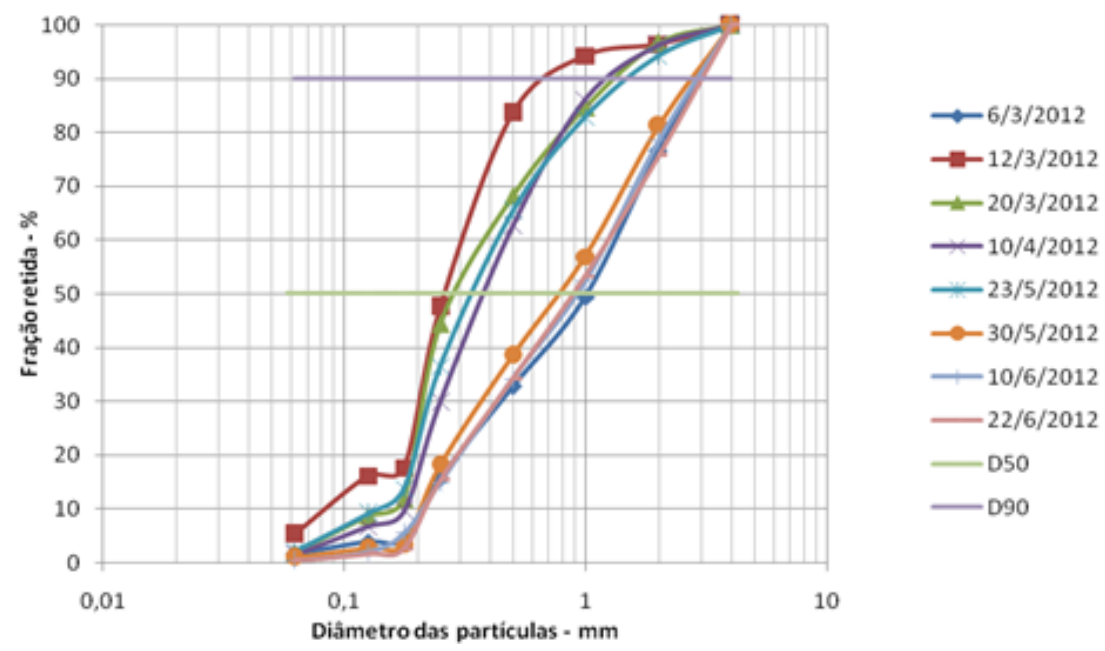

Figura 3: Curvas granulométricas do material de leito

Já a pesquisa realizada por Souza (2011) na bacia hidrográfica do Rio Capibaribe, em que compreendeu a maior parte a classe textural de areia grossa e em menor parte muito grossa e 
areia média, segundo a classificação adotada pela American Geophysical Union (AGU), para os níveis de vazões obtidos.

Essa distribuição do material de leito tem sido bastante investigada por diversos autores que procuraram avaliar muitas vezes a sua variação ao longo do curso do Rio. Cangani et al. (2008), trabalhando no Rio Alto Paraná constatou que a distribuição do diâmetro médio dos sedimentos transportado nos canais, sofre pouca variação, encontrando valores entre 0,125 a 0 , $250 \mathrm{~mm}$.

A Tabela 3 apresenta os valores de d50, d90 das partículas de sedimentos obtidos da Figura 3, os valores do coeficiente de Manning ( $n$ ) da seção referentes às diversas campanhas, determinados empregando-se os valores de $\mathrm{d} 50$ e $\mathrm{d} 90$, bem como os valores do gradiente de energia (I), coeficiente de rugosidade do leito (ks), coeficiente de rugosidade das partículas (Kr) e descarga sólida do leito $-\mathrm{t} / \mathrm{s}$ por $\mathrm{m}$ de largura $\left(\mathrm{q}_{\mathrm{sa}}\right)$.

Tabela 3: Valores de $\mathrm{d} 50$ e $\mathrm{d} 90$ das partículas de sedimentos e de $\mathrm{n}$ de Manning (em $\mathrm{mm}$ )

\begin{tabular}{c|c|c|c|c|c|c|c|c|c}
\hline Data & $\mathbf{d 5 0}$ & $\mathbf{d 9 0}$ & $\mathbf{n 5 0}$ & $\mathbf{n 9 0}$ & $\mathbf{n}$ & $\mathbf{I} \mathbf{( m / m})$ & $\mathbf{K s}$ & $\mathbf{K r}\left(\mathbf{m}^{\mathbf{1 / 3}} \mathbf{/ s}\right)$ & $\mathbf{q}_{\mathbf{s a}}(\mathbf{t} / \mathbf{m s})$ \\
\hline $6 / 3 / 2012$ & 1,000 & 3,000 & 0,047 & 0,046 & 0,047 & $3,80 \mathrm{E}-04$ & 21,379 & 21,649 & $5,79 \mathrm{E}-05$ \\
\hline $12 / 03 / 12$ & 0,260 & 0,640 & 0,038 & 0,036 & 0,037 & $1,37 \mathrm{E}-04$ & 27,169 & 28,008 & $1,44 \mathrm{E}-05$ \\
\hline $20 / 03 / 12$ & 0,280 & 1,400 & 0,038 & 0,041 & 0,040 & $1,30 \mathrm{E}-04$ & 25,305 & 24,582 & $1,12 \mathrm{E}-05$ \\
\hline $10 / 04 / 12$ & 0,380 & 1,200 & 0,040 & 0,040 & 0,040 & $8,51 \mathrm{E}-05$ & 24,999 & 25,222 & $2,97 \mathrm{E}-06$ \\
\hline $23 / 05 / 12$ & 0,350 & 1,500 & 0,040 & 0,041 & 0,040 & $7,47 \mathrm{E}-05$ & 24,706 & 24,301 & $2,60 \mathrm{E}-06$ \\
\hline $30 / 05 / 12$ & 0,690 & 2,800 & 0,045 & 0,046 & 0,045 & $1,05 \mathrm{E}-04$ & 22,175 & 21,899 & $1,39 \mathrm{E}-06$ \\
\hline $10 / 06 / 12$ & 0,850 & 2,900 & 0,046 & 0,046 & 0,046 & $1,07 \mathrm{E}-04$ & 21,732 & 21,772 & $6,71 \mathrm{E}-08$ \\
\hline $22 / 06 / 12$ & 0,820 & 3,000 & 0,046 & 0,046 & 0,046 & $1,02 \mathrm{E}-04$ & 21,736 & 21,649 & $6,92 \mathrm{E}-08$ \\
\hline
\end{tabular}

É de esperar-se que os sedimentos mais grossos ocorram em momentos de vazões mais altas, com maior capacidade de arraste, contudo, ocorrem os mesmos comportamentos para as maiores e menores vazões observadas. Também foi observado por Souza (2011), no estudo realizado na bacia hidrográfica do Rio Capibaribe, que a variação de transporte de sedimento de fundo foi relacionada com uma variação na descarga líquida, sendo para níveis de variação de vazão de 0,25 a $11,60 \mathrm{~m}^{3} \mathrm{~s}^{-1}$, correspondeu a uma variação na descarga sólida de sedimento de fundo de 0,20 e 3,41 tdia ${ }^{-1}$, respectivamente.

Esse comportamento pode estar associado às ações de retirada de material do leito do rio junto a ponte, alterando as condições naturais do leito, causando modificação na distribuição granulométrica do material do leito, tendo como consequência a modificação do coeficiente de rugosidade do leito e das partículas na seção do rio, o qual é justamente função da dimensão característica das partículas. Outro fator que também pode estar relacionado com a construção de barramentos no rio, como mostrou o estudo realizado por Verstraeten e Prosser (2008) na Bacia do Rio Murrumbidgge (Austrália) ao constatarem redução de $47 \%$ na descarga de sedimento após a instalação de barramentos no rio.

A equação de Meyer-Peter e Müller produziu resultados que aparentemente seguem a variação da granulometria dos sedimentos do leito do rio, apresentando valores que variaram de acordo com a descarga líquida, contudo apresenta discrepância de valores de descarga sólida de fundo para valores próximos de vazão. 


\section{CONCLUSÕES}

O uso da equação de Meyer-Peter \& Müller para a estimativa do transporte de sedimentos no rio pode não ter uma precisão adequada, possivelmente devido a problemas na calha do rio com a retirada de materiais. No entanto, foi possível mensurar a descarga sólida de leito do rio Piranhas dentro de valores bem aceitáveis.

Os tipos de materiais sólidos que foram carreados e/ou depositados no Rio Piranhas durante o período de estudo, foram constituídos de pedregulho a areia fina.

Recomenda-se o prosseguimento do estudo, aplicando-se outros modelos e medições de campo para melhores comparações e validações de equações aplicadas.

\section{REFERÊNCIAS}

1. ANA. Agência Nacional de águas. Instruções de Operação - Rede Hidrometeorológica. 2011. Agência Nacional de Águas, Brasília, p. 1-17.

2. APITZ S.E.; WHITE S.M. A conceptual framework for river-basin-scale sediment management, Journal of Soils and Sediments, v.3, n. 3, p.132-138, 2003.

3. CANGANI, M. T.; POLEGATTO, J. C.; ROCHA, P. C. Análise granulométrica de sedimentos de fundo nos canais de planície fluvial do Rio Alto Paraná. In: Fórum Ambiental da Alta Paulista. v.5, p.23-28, 2008.

4. CARVALHO, N. de O.; FILIZOLA JÚNIOR, N. P.; SANTOS, P. M. C. dos; LIMA, J. E. F. W. Guia de Práticas Sedimetométricas. Agência Nacional de Energia Elétrica, Superintendência de Estudos e Informações Hidrológicas, ANEEL, Setembro, 2000, Brasília, DF.

5. CARVALHO, N. O.; SALGADO, J. C. M.; ROSS, J.; RUIZ, N. C. P.; SILVA, L. F. A., MESQUITA, J. B.; GOULART NETTO, A. A.; SILVA, L. P. Revisão dos estudos sedimentológicos do rio Madeira e Mamoré, RO. In: XVI Simpósio Brasileiro de Recursos Hídricos, Novembro, 2005, João Pessoa, PB.

6. CARVALHO, N. de O. Hidrossedimentologia Prática. 2a Ed., rev., atual. e ampliada- Rio de Janeiro: Interciência, 2008.

7. COBANER, M.; UNAL, B.; KISI, O. Concentration estimation of sediment suspended by an adaptive neuro-fuzzy and neural network approaches using hydro-meteorological data, J. of Hydrology, v.367, n. 1-2, p.52-61, 2009.

8. SOUZA, W. L. da S. Produção de sedimentos da bacia hidrográfica do rio Capibaribe para zona costeira da região metropolitana do Recife. Recife, 2011. 136f. Dissertação (Mestrado em Ciência do Solo). Universidade Federal Rural de Pernambuco.

9. VERSTRAETEN, G.; POESEN, J. Factors controlling sediment yield from small intensively cultivated catchments in a temperate humid climate. Geomorphology, n. 40, p.123-144, 2001. 